\title{
Studying Stress on e-Learning Users
}

\author{
Davide Carneiro, Sérgio Gonçalves, Paulo Novais, and José Neves \\ CCTC/Department of Informatics, University of Minho \\ Braga, Portugal \\ \{dcarneiro, pjon, jneves\}@di. uminho.pt \\ sergi omcgoncal ves@gmail .com\}@springer . com
}

\begin{abstract}
E-Learning, much like any other communication processes, has been significantly shaped by technological evolution. In its original form, e-Learning aimed to bring the education closer to people, making it more modular and personalized. However, in reality, we observe that it represents a separation between student and teacher, simplifying this relationship to the exchange of "text-based messages", leaving aside all the important contextual richness of the classroom. We are addressing this issue by devising a contextual layer for e-Learning platforms. Particularly, in this paper we describe a solution to convey information about the level of stress of the students so that the teacher can take better and more informed decisions concerning the management of the learning process.
\end{abstract}

Key words: Stress, Behavioural Analysis, Moodle

\section{Introduction}

In traditional teaching, the relationship between teachers and students is a close one. The almost daily access to the teacher results in a sense of proximity, of accompaniment: they feel that the teacher cares. This is essential for the student's motivation. Teachers, on the other hand, benefit from this proximity by having a constant update on the state of the students, on their worries, on their feedback concerning each topic. All this contextual information, much of it analysed in an unconscious way, allows the teacher to intuitively assess his students and steer his methodologies and strategies in order to optimize success.

However, when a student attends an electronic course or makes use of an e-Learning platform, the interaction between student and teacher, without all these non-verbal interactions, is poorer. Thus, the assessment of the feelings, the state and the attitudes of the student by the teacher becomes more difficult. In that sense, the use of technological tools for teaching, with the consequent teacher-student and student-student separation, may represent a risk as a significant amount of context information is lost. Since students' effectiveness and success in learning is highly related and can be partially explained by their mood while doing it, such issues should be taken into account when in an e-Learning environment. In a traditional classroom, the teacher can detect and even foresee 
that some negative situation is about to occur and take measures accordingly to mitigate it. When in a virtual environment, similar actions are impossible.

The lack of certain aspects of communication in Virtual Environments (VEs) that include our body language, tone of voice or gestures has been studied by research (see for example [1-4]). The general lesson to learn from here is that human communication is a rich multi-modal process that cannot, by any means, be reduced to simple words without a significant loss in performance [5].

Hover, stress and emotions, in particular, can play an important (usually negative) role in education $[7,9]$. In that sense, its analysis in an e-Learning environment assumes greater importance. Generally, stress assessment is done either through questionnaires (an easily misleading approach and certainly not a dynamic one) or through physiological sensors (very accurate but invasive to the point of making them impractical in e-Learning).

In [10], the authors studied emotion in users of e-Learning platforms. They do it using four physiological sensors: hearth rate, skin conductance, blood volume pressure and EEG brainwaves. Despite its accuracy, this approach will never result in a real-life application due to its invasiveness. Other less invasive approaches also exist. [6] contains an overview of different applications of the socalled Affective Computing [8] field in e-Learning. They point out the use of facial expression and speech recognition as potential ways to detect emotional states. However, facial recognition requires a dedicated camera of some quality to be placed in front of the user otherwise it will be inaccurate. It is thus also invasive. Speech recognition, on the other hand, is less invasive but is also much more prone to error, being difficult to develop an accurate speech model given that each individual has his own speech rhythm, tone, pitch or intonation, aspects that are much cultural-dependent.

With these issues in mind, in this paper we present a novel approach to assess the level of stress of students on an e-Learning platform. It is characterized by being non-invasive, personal and transparent. Our objective is indeed to assess the level of stress of students by analysing their behaviour when using the eLearning platform, i.e., their interaction patterns while using the mouse and the keyboard. A total of 12 features are extracted and analysed that fully describe the way students use these peripherals when under the effect of stress.

\section{Experimental study}

In order to analyse the effect of stress on the use of such platforms, we studied specifically the behaviour of students while performing evaluation activities. In this section it is described the experimental study that was conducted with this aim, involving 74 students.

During the process of data collection, two scenarios were set up. In Scenario A, an activity was performed whose main objective was simply to assess the student's knowledge on the selected topics, with no effect on their marks. The activity was performed without any constraints; the students were simply requested to answer the questions that were provided on Moodle. 
In a posterior phase, the students were requested to participate on Scenario B. Under this scenario they had to perform another activity, now with some constraints. The students were told by the teacher that this activity would be effectively used for their evaluation, with an impact on the final score. They were also given access passwords and the notion that they would be responsible for managing their activity to maximize their score given the time available. While the students were performing the activity, the teacher insisted regularly on the importance of their performance on their final score and on the decreasing amount of time available.

When analysing the behaviours of the students during the execution of the activities under the two scenarios, the teacher noted that the students were visibly more stressed on the second scenario. After brief talk with some of them, their main feedback concerned their disappointment for not completing the activity or for not answering each question with the needed attention. The most frequent factors for the feeling of unnacomplishment included: (1) the time limit; (2) the noise on the room; (3) the constant interventions of the teacher; (4) the existence of a password for initiating the activity; and (5) the weight of their performance on the final score.

Despite the two activities being very similar in terms of contents and difficulty, it was observed by the teacher that, for similar questions, students would often answer incorrectly or in an incomplete way.

\subsection{Methodology and Data Collection}

Six different classes participated in the study, which resulted in 12 different moments of data collection: one for each class and for each scenario. The study involved a total of 74 students, 49 boys and 25 girls, aged between 13 and 17 .

The data gathered was analysed in order to determine statistically significant differences between scenarios A and B. This analysis was performed with the data of each student individually and with all the data collected. Measures of central tendency and variability were calculated for all variables of interest. Provided that most of the distributions are not normal, the Mann-Whitney-Wilcoxon Statistical test was used to test whether there are actual differences between the distributions of the data for the two scenarios. The data analysis was performed using Wolfram Mathematica, Version 8.0.

In order to collect the data used for analysing the behaviour of the students a very simple logger was developed that was kept running throughout the duration of the activities. This logger was not visible for the student and had no effect on the usability of the Moodle platform. The main objective of the logger was to collect data on how, in each of the activities, the student used the mouse and the keyboard, for later analysis.

The logger listens to system events concerning the mouse and the keyboard. It generates a list of the following events:

- MOV, timestamp, posX, posY - an event describing the movement of the mouse, in a given time, to coordinates (posX, posY); 
- MOUSE_DOWN, timestamp, [Left|Right], posX, posY - this event describes the first half of a click (when the mouse button is pressed down), in a given time. It also describes which of the buttons was pressed (left or right) and the position of the mouse in that instant;

- MOUSE_UP, timestamp, [Left|Right], posX, posY - an event similar to the previous one but describing the second part of the click, when the mouse button is released;

- MOUSE_WHEEL, timestamp, dif - this event describes a mouse wheel scroll of amount dif, in a given time;

- KEY_DOWN, timestamp, key - describes a given key from the keyboard being pressed down, in a given time;

- KEY_UP, timestamp, key - describes the release of a given key from the keyboard, in a given time;

A different $\log$ is built for each student under each scenario. The data collected allows to build information about the following features:

- Key Down Time - the timespan between two consecutive KEY_DOWN and KEY_UP events, i.e., for how long was a given key pressed (in milliseconds).

- Time Between Keys - the timespan between two consecutive KEY_UP and KEY_DOWN events, i.e., how long did the individual took to press another key (in milliseconds).

- Velocity - The distance travelled by the mouse (in pixels) over the time (in milliseconds). The velocity is computed for each interval defined by two consecutive MOUSE_UP and MOUSE_DOWN events.

- Acceleration - The velocity of the mouse (in pixels/milliseconds) over the time (in milliseconds). A value of acceleration is computed for each interval defined by two consecutive MOUSE_UP and MOUSE_DOWN events.

- Time Between Clicks - the timespan between two consecutive MOUSE_UP and MOUSE_DOWN events, i.e., how long did it took the individual to perform another click (in milliseconds).

- Double Click Duration - the timespan between two consecutive MOUSE_UP events, whenever this timespan is inferior to 200 milliseconds.

- Average Excess of Distance - this feature measures the average excess of distance that the mouse travelled between each two consecutive MOUSE_UP and MOUSE_DOWN events (in pixels).

- Average Distance of the Mouse to the Straight Line - in a few words, this feature measures the average distance of the mouse to the straight line defined between two consecutive clicks (in pixels).

- Distance of the Mouse to the Straight Line - this feature is similar to the previous one in the sense that it will compute the distance to the straight line between two consecutive MOUSE_UP and MOUSE_DOWN events. However, it returns the sum rather than its average value during the path (in pixels). 
- Signed Sum of Angles - with this feature the aim is to determine if the movement of the mouse tends to "turn" more to the right or to the left (in degrees).

- Absolute Sum of Angles - this feature is very similar to the previous one. However, it seeks to find only how much the mouse "turned", independently of the direction to which it turned.

- Distance between clicks - represents the total distance travelled by the mouse between two consecutive clicks, i.e., between each two consecutive MOUSE_UP and MOUSE_DOWN events.

\section{Results}

Here, a detailed feature-by-feature analysis is performed considering the data collected from all the participants. This aims to identify behaviours that are, with a significant measure of support, common to all the participants. Given the length of the features, only the most interesting ones were selected.

In order to perform this analysis we made a prior individual analysis in which we computed the mean and median value of each feature, for each individual. These values were then combined into a single dataset and analysed together.

Key Down Time When analysing the average time that a key is pressed down while typing, the main conclusion is that a stressed student tends to press the keys during a smaller amount of time. While on the baseline data the mean duration of this feature is of $102.85 \mathrm{~ms}$, under stress the mean value is of 97.8 ms. This same trend was observed in $70.5 \%$ of the students. Concerning the median, it decreases in average from $98.5 \mathrm{~ms}$ to $96.2 \mathrm{~ms}$, showing a decreasing tendency in $68.9 \%$ of the cases analysed. However, this does not necessarily indicates that the student writes faster when under stress, only that he spends less time pressing the keys.

When observing the significance of the differences between the baseline and stressed distributions, for each student, only in $31 \%$ of the cases are the differences statistically significant. However, the trend of decreasing time while the key is down does exist.

Time Between Keys In this feature the time spent between pressing two consecutive keys is analysed, which defines the typing velocity. While without stressors, a student spends in average nearly 3 seconds between each two consecutive keys pressed $(2904.86 \mathrm{~ms})$. While under stress, this time increases to $5202.19 \mathrm{~ms}$. Moreover, $60 \%$ of the students evidence this increase in the average and $83.6 \%$ evidence an increase in the median, from $449.156 \mathrm{~ms}$ in average to $1979.51 \mathrm{~ms}$.

This denotes that the student writes at a slower pace, when under stress. Statistically significant differences between the baseline and the stressed data for each student were observed in $54 \%$ of the cases. 
Fig. 1. The time (in milliseconds) during which a key remains down while typing tends to decrease when students are under stress.

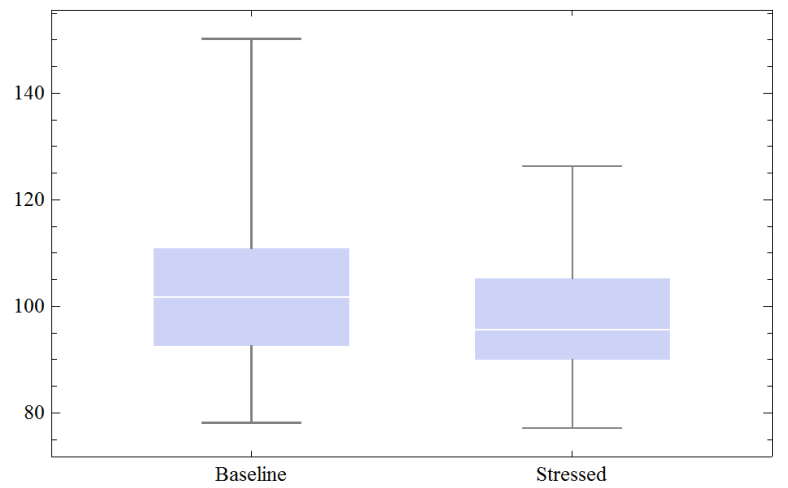

Fig. 2. The time spent between each two consecutive keys being pressed tends to increase when under stress.

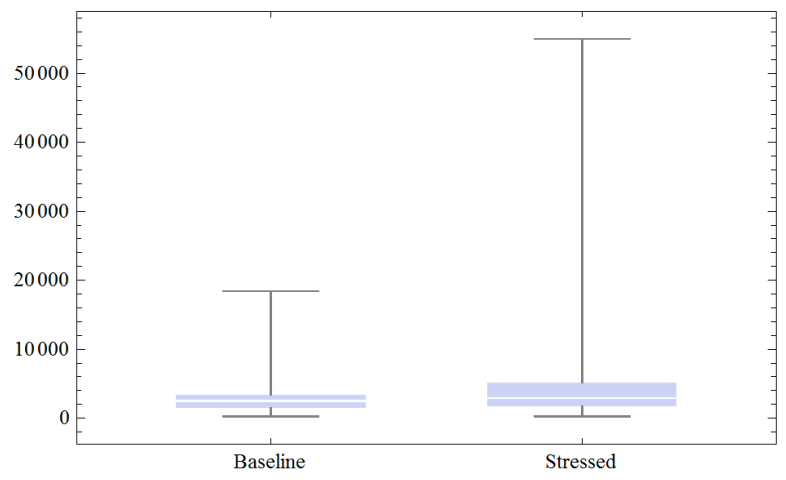

Acceleration The initial expectation was that when under stress, students would have faster and more sudden movements, sometimes even involuntary or arbitrary. However, the results point the other way around: the acceleration measured on the mouse is smaller when the students are under stress.

The mean value of the acceleration between each two consecutive clicks in the baseline data is $0.532 \mathrm{px} / \mathrm{ms}^{2}$, decreasing to $0.449 \mathrm{px} / \mathrm{ms}^{2}$, which represents a difference of $-0.083 \mathrm{px} / \mathrm{ms}^{2}$. This decreasing tendency in the mean value of the acceleration was observed in $77.42 \%$ of the students. If we consider the value of the median of the acceleration, it is of $0.2 \mathrm{px} / \mathrm{ms}^{2}$ in the baseline data and of $0.169 \mathrm{px} / \mathrm{ms}^{2}$ in the stressed data. $87.1 \%$ of the students evidence a decrease in the median of the acceleration. This points out a remarkable tendency that can be generalized to a large number of students.

Concerning the statistical significance of this data, significant differences between the baseline and the stressed data have been observed in $77 \%$ of the 
Fig. 3. Two box plots detailing the differences on the acceleration between the baseline and stressed data. In general, stressed students have smaller values of acceleration.

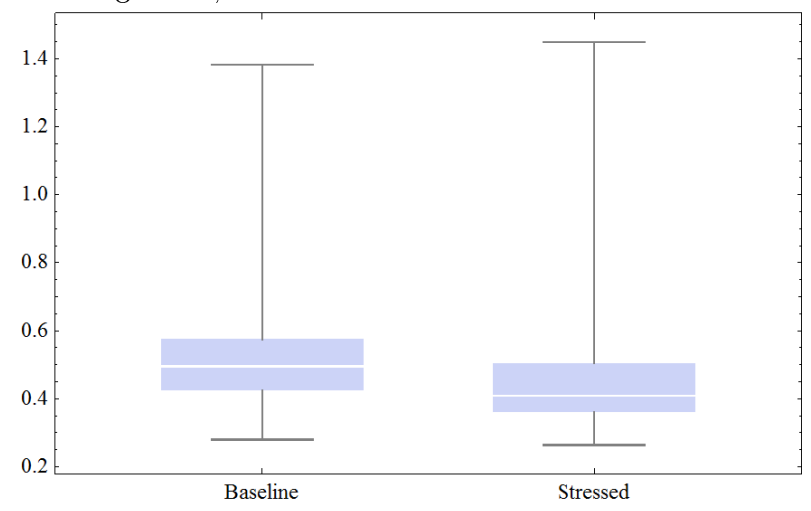

Fig. 4. Similarly to the acceleration, the value of the velocity of the mouse decreases with stress.

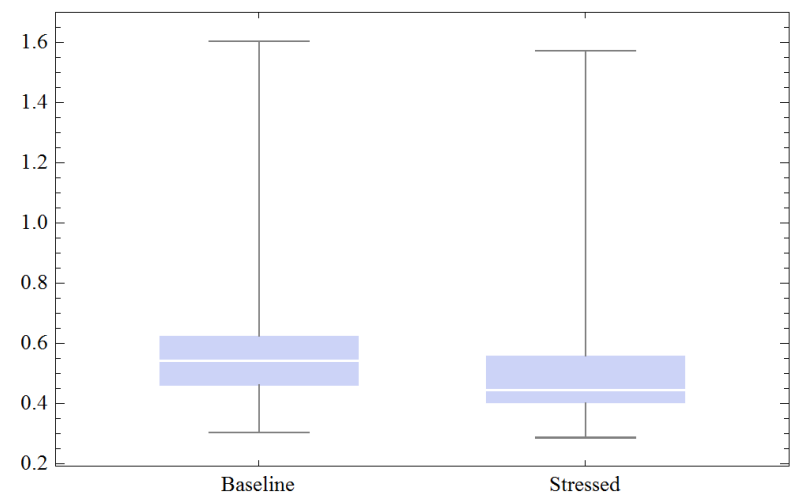

students, i.e., not only were they affected but the differences due to the effects were statistically significant.

Velocity Similarly to acceleration, an increase in the velocity was expected due to stress. However, the opposite tendency was observed: stressed students move their mouse slower. A decrease in the mean value of the velocity between each two clicks was observed in $77.4 \%$ of the students, from $0.58 \% \mathrm{px} / \mathrm{ms}$ to $0.49 \mathrm{px} / \mathrm{ms}$. The difference in the median was even more striking, decreasing in $90.3 \%$ of the students, from $0.22 \mathrm{px} / \mathrm{ms}$ to $0.189 \mathrm{px} / \mathrm{ms}$. Similarly to acceleration, a large number of students showed this same tendency. Moreover, significant statistical differences between the calm and the stressed data have been observed in $81 \%$ of the students. 
Fig. 5. Two box plots detailing the differences on the time spent between clicks, for the baseline and stressed data. Generally, stressed students spend less time between clicks.

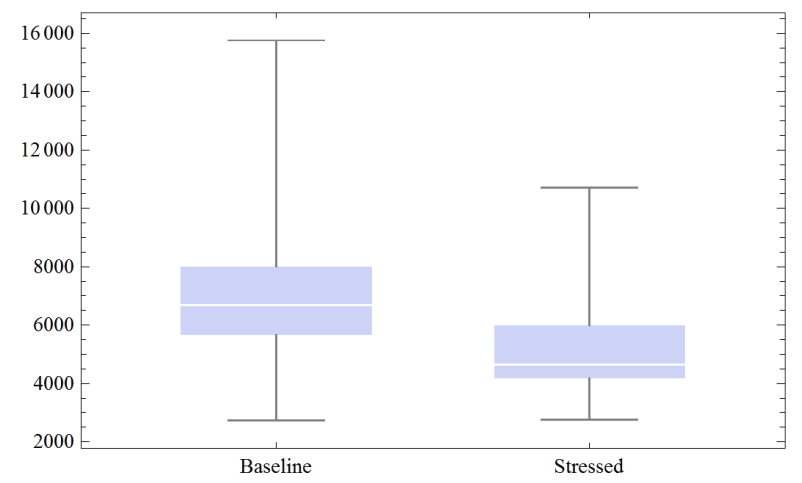

Time Between Clicks The rate at which the students use the mouse to click is affected by stressors and a marked tendency can also be identified: when under stress, students spend less time between each consecutive click. While without stressors each student spends roughly 7 seconds without clicking (7033 ms), under stress this time decreases nearly 2 seconds to $5104 \mathrm{~ms}$. This tendency of decreasing was observed in $80.6 \%$ of the students. Concerning the median, its value is of $3149.18 \mathrm{~ms}$ for the baseline data, decreasing to 2349.61 on the stressed data. The median decreases in $74.2 \%$ of the cases.

However, concerning the significance of the differences for each student, only $32 \%$ of the students evidence statistically significant differences between the baseline and stressed data. This points out that, although the tendency does exist, it might not be a such marked one.

Average Distance of the Mouse to the Straight Line The average distance of the pointer to the straight line defined by two consecutive clicks also tends to decrease with stress, meaning that stressed students become more efficient in the way they move the mouse, moving in more straight lines between their objectives. The mean value of this feature for all students while without stressors was of 59.85 pixels, decreasing to 44.51 pixels when under stress, a decrease of $25.63 \%$ in the average distance. $85.48 \%$ of the students evidence this same behaviour. Similarly, the median decreases for $82.26 \%$ of the students, from and average of 30.14 to 16.63 pixels.

Distance of the Mouse to the Straight Line This feature is related to the previous one, expect that it measures the total excess of the distance to the straight line between each two clicks, rather than its average value. Thus being, the results observed are in line with the previous ones. The sum of the distances of the mouse to the closest point in the straight line between the two clicks is in 
Fig. 6. These two box plots show that when a student is stressed, he moves the mouse with more precision as he minimizes the distance to the straight line that is defined by each two consecutive clicks.

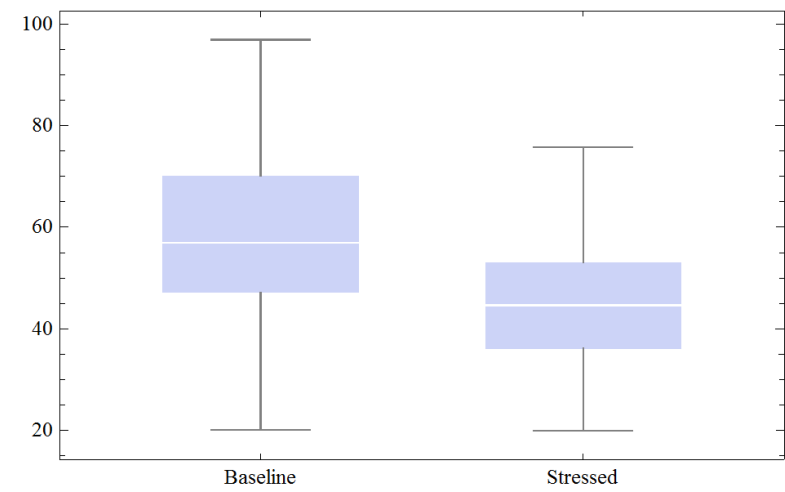

Fig. 7. These two box plots allow to reach a conclusion in line with the previous feature.

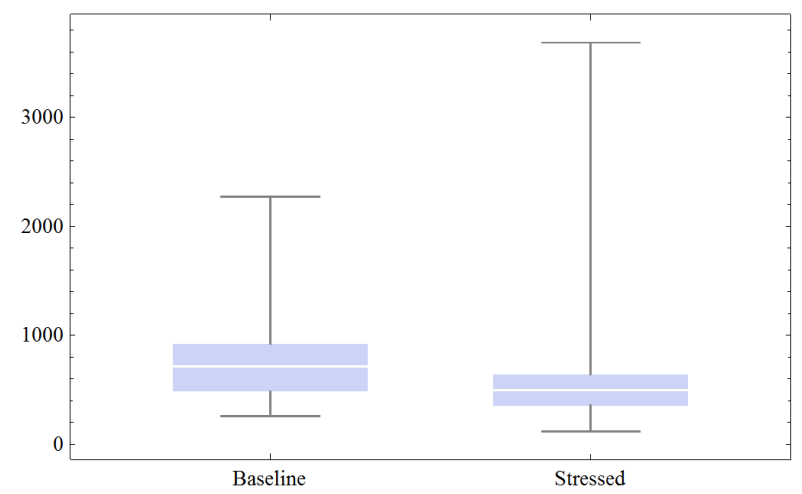

average 782.03 pixels without stressors, decreasing to 549.752 pixels when under stressors. $87.1 \%$ of the students behave like this while under stress. The value of the median also decreases in average from 241.1 pixels to 104.07 pixels, with $80.65 \%$ of the students showing a decrease in its value.

Absolute Sum of Angles Here we analysed the absolute sum of angles, i.e., "how much" the mouse turned rather than "to which side" the mouse turned more. Without stress, between each two clicks the mouse turned in average $8554.4^{\circ}$, while under stress this value decreased to $5119-75^{\circ}$, which represents a decrease of $764.64^{\circ}$ between each two clicks, in average. Moreover, $69.35 \%$ of the students decrease the amount of how much they turn their mouses. The value of the median also decreases from $6598.54^{\circ}$ to $3134.04^{\circ}$. 
Fig. 8. These box plots show that, in line with previous conclusions, stressed students move in a more straight line, curving less.

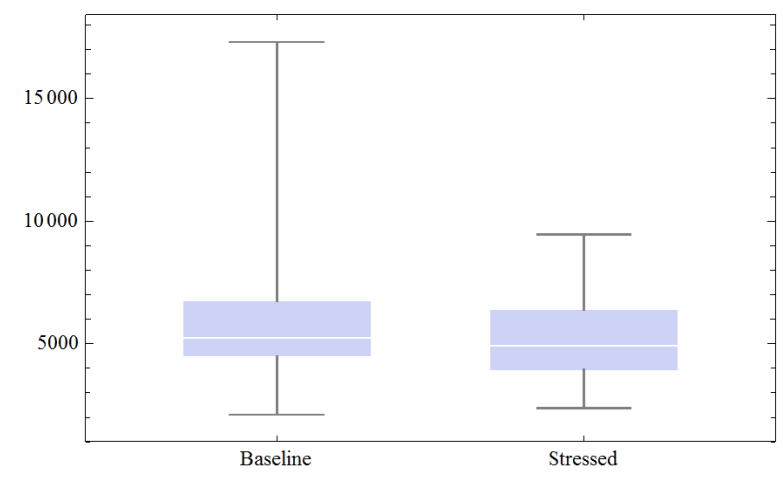

Fig. 9. These plots show that, despite some outlier, students tend to move their mouse less when they are under stress: they become more precise.

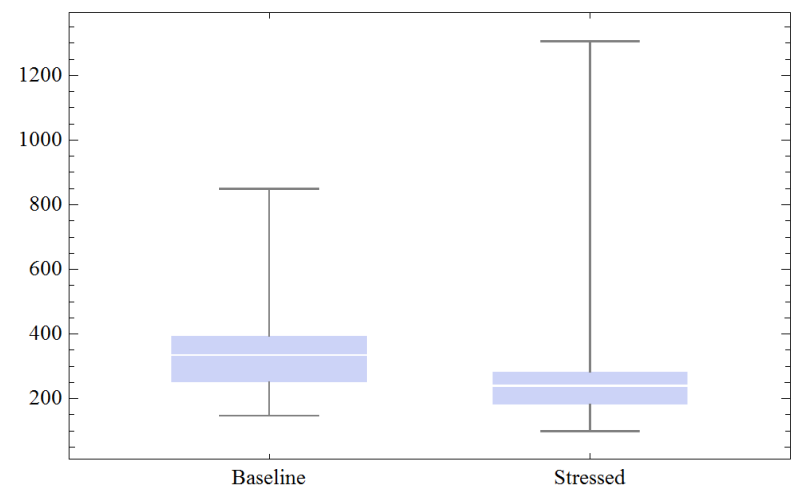

Distance Between Clicks The total distance travelled by the mouse between each two consecutive clicks also shows a consistent decrease due to stress. In average, the mouse of a student that was not stressed travelled 342.61 pixels between each to consecutive clicks. This value decreased by 92 pixels to 250.64 pixels while under stress, a decrease of $27 \%$, in average. $85.5 \%$ of the students evidence this behaviour. The median value also decreases, for $87.1 \%$ of the students, from 186.5 to 103.5 pixels.

\section{Conclusion and Future Work}

This paper started by analysing current drawbacks of online communication tools, specifically in the context of e-Learning. Interestingly enough, e-Learning tools, that started with the objective of bringing teacher and students closer together, actually end up having the opposite effect. The teacher may even be 
more accessible, easily reachable on the other end of the screen. However, there is also a significant sense of distance that is nowadays hard to overcome.

Besides other issues identified such as lack of motivation by the part of the students, what we are really concerned is for the lack of important contextual information. Generally, when a teacher analysis the state of his students, he takes into consideration more information than just the evaluation results. He builds, partially in an intuitive way, a representation of the student that includes the subjects that he is more comfortable at, a measure of this degree of comfort, among other issues. And he does so by having a daily access to pieces of information such as the attitudes, the actions, the reactions, the behaviours inside and outside the classroom or the occasional small-talk. All this important information is absent from an e-Learning platform. The teacher must thus assess the state of his students merely by the results of the activities performed, which is a far more poor approach.

In this paper we detailed an approach that aims to bridge this gap by providing the teacher with real-time access to context information about his students. Particularly, we have focused on the study of stress as this is one of the key factors that influence the performance of the student.

The main conclusion achieved is that students, when under stress, tend to behave in a more efficient way, decreasing the number of unnecessary actions: they are more focused on their task. However, some students were also found that behave the other way around: they become less efficient and more sloppy when under stress. This points out that: 1) generic models can be developed that can, to a large extent, shape the response of students to stress; and 2) personalized models should not be disregarded as stress is a very individual phenomenon, with each student having his own particular response.

The results achieved show not only that stress does have an effect on the interaction patterns and also that this influence can be quantified and measured. Nevertheless, the features related with the mouse show much more satisfying results than the ones related with the keyboard. We assume that this can be related with the nature of the experiment: students were not required to write much, they mostly had to click on the correct answers. The data collected from the mouse is thus much more than the data collected from the keyboard. In future work we will study the use of the keyboard in more detail in order to build a more detailed stress model.

We are also now starting a similar study to measure the effects of fatigue on the use of the same peripherals. The main goal of this study is, similarly, to determine if the effects of fatigue can be measured and quantified. Our ultimate goal is to develop multi-modal non-invasive classifiers that can act in real time to provide the best and most accurate description of the state of the student to the teacher. This will bring the students closer to the teacher, allowing him to better understand their difficulties and the topics in which they are more at ease. Ultimately, with access to this information, the teacher will be able to adjust his teaching strategies and methodologies to shape such changes, making the learning process a more efficient one. 
Acknowledgments. This work is part-funded by ERDF - European Regional Development Funds through the COMPETE Programme (operational programme for competitiveness) and by National Funds through the FCT - Fundação para a Ciência e a Tecnologia (Portuguese Foundation for Science and Technology) within project FCOMP-01-0124-FEDER-028980 (PTDC/EEI-SII/1386/2012). The work of Davide Carneiro is also supported by a doctoral grant by FCT (SFRH/BD/64890/2009).

\section{References}

1. Alsina-Jurnet, I., Gutiérrez-Maldonado, J.: Influence of personality and individual abilities on the sense of presence experienced in anxiety triggering virtual environments. International Journal of Human-Computer Studies, 68(10):788-801 (2010)

2. Beale, R., Creed, C.: Affective interaction: How emotional agents affect users. International Journal of Human-Computer Studies, 67(9):755-776 (2009)

3. Dodds,T.J., Mohler, B.J., Bülthoff, H.H.: Talk to the Virtual Hands: Self-Animated Avatars Improve Communication in Head-Mounted Display Virtual Environments. PLoS ONE, 6(10):e25759 (2011)

4. Hudlicka, E.: To feel or not to feel: The role of affect in human-computer interaction. International Journal of Human-Computer Studies, 59(1-2):1-32 (2003)

5. Jaimes, A., Sebe, N.: Multimodal human-computer interaction: A survey. Computer Vision and Image Understanding, 108(1- 2):116-134 (2007)

6. Lin, H., Pan, F., Wang, Y., Lv, Sv., Sun, S.: Affective Computing in E-learning, E-learning, InTech, ISBN 978-953-7619-95-4, 118-128 (2010)

7. Palmer, S., Cooper, C., Thomas, K.: Creating a Balance: Managing Stress, London: British Library (2003)

8. Picard, R. W. Affective Computing. The MIT Press (2000)

9. Rodrigues, M., Fdez-Riverola, F., Novais, P.: Moodle and Affective Computing Knowing Who's on the Other Side, ECEL-2011 - 10th European Conference on E-learning, ISBN: 978-1-908272-22-5, pp. 678-685, Brighton, UK (2011)

10. Shen, L., Wang, M., Shen, R.: Affective e-Learning: Using "Emotional" Data to Improve Learning in Pervasive Learning Environment, Educational Technology \& Society, 12 (2), 176-189 (2009) 